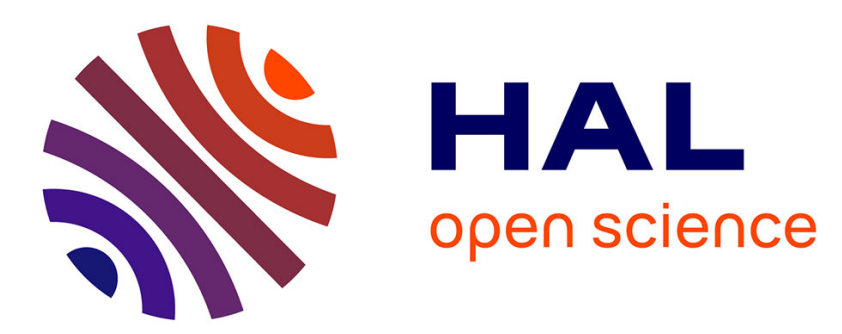

\title{
Regulation of Secondary metabolism in the gray mold fungus Botrytis cinerea
}

\author{
Muriel M. Viaud, Antoine Porquier, Adeline A. Simon, Julia Schumacher
}

\section{To cite this version:}

Muriel M. Viaud, Antoine Porquier, Adeline A. Simon, Julia Schumacher. Regulation of Secondary metabolism in the gray mold fungus Botrytis cinerea. Host-Pathogen Interaction. Microbial Metabolism, Pathogenicity and Antiinfectives, 6, Wiley-VCH, 450 p., 2016, Drug Discovery in Infectious Diseases, 978-3-527-68239-3. hal-02795159

\section{HAL Id: hal-02795159 \\ https://hal.inrae.fr/hal-02795159}

Submitted on 5 Jun 2020

HAL is a multi-disciplinary open access archive for the deposit and dissemination of scientific research documents, whether they are published or not. The documents may come from teaching and research institutions in France or abroad, or from public or private research centers.
L'archive ouverte pluridisciplinaire HAL, est destinée au dépôt et à la diffusion de documents scientifiques de niveau recherche, publiés ou non, émanant des établissements d'enseignement et de recherche français ou étrangers, des laboratoires publics ou privés. 
․ Edited by

$3 \rightarrow$ Gottfried Unden, Eckhard Thines, and Anja Schüffler

Host -

Pathogen Interaction

Microbial Metabolism, Pathogenicity and Antiinfectives

Volume 6

Series Editor:

Paul M. Selzer

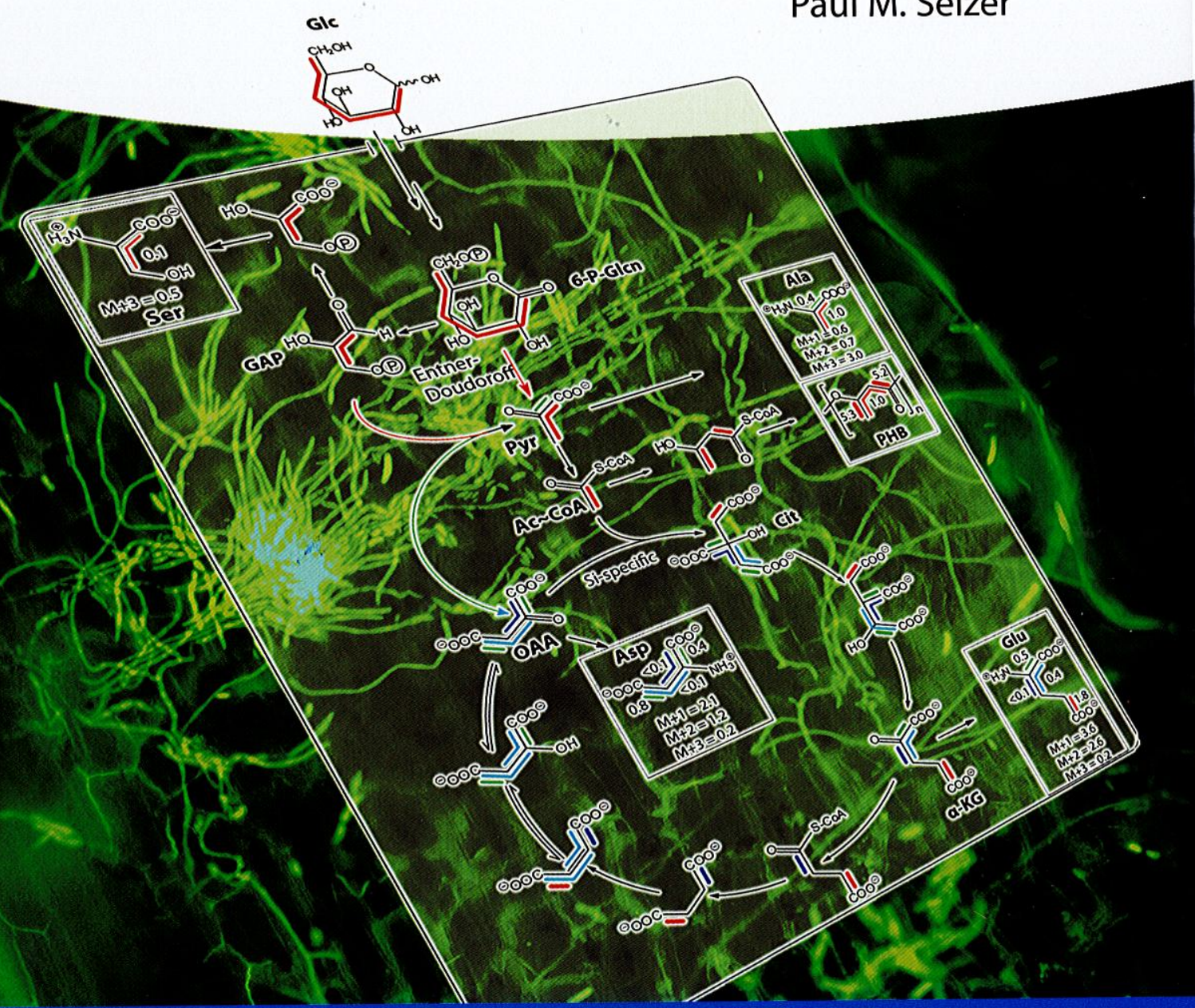

Drug Discovery in Infectious Diseases 


\title{
11
}

\section{Regulation of Secondary Metabolism in the Gray Mold Fungus Botrytis cinerea}

Muriel Viaud*, Julia Schumacher, Antoine Porquier, and Adeline Simon

\begin{abstract}
Botrytis cinerea is responsible of the gray mold disease on a wide range of host plants. During the infection process, it produces unspecific phytotoxins (botrydial and botcinic acid) and other secondary metabolites that play a significant role in plant tissue colonization. Regulation of the biosynthesis of these bioactive compounds is therefore crucial for the outcome of fungus/plant interactions. Combining genetics and transcriptomic approaches, the role of different signaling cascades, transcription factors, and other regulators such as those belonging to the VELVET complex have recently been investigated. Overall, these studies suggest a strong link between the regulation of secondary metabolism and the light-dependent development in B. cinerea. Further knowledge of the complex regulatory network controlling secondary metabolism in $B$. cinerea is crucial to understand the interaction between $B$. cinerea and its different host plants and may help to define new strategies to control this crop-devastating fungus.
\end{abstract}

\section{Botrytis cinerea, the Causal Agent of Gray Mold Disease}

Botrytis cinerea Pers. Fr. causes severe pre- and postharvest crop losses on more than 200 host plants including important crops and is therefore one of the most studied phytopathogenic fungi [1]. This Ascomycete (Leotiomycetes class, Sclerotiniaceae family) is also named the gray mold agent because of the production of masses of melanized conidia on infected plants. Conidia allow $B$. cinerea to disperse widely on further plants while the ability to form sclerotia allows the fungus to survive in the soil for extended periods. These melanized survival structures can also be fertilized by microconidia from a strain of the opposing mating type to form fruiting bodies (apothecia) with sexual recombinants (ascospores) [2,3].

B. cinerea is considered a typical necrotroph, inducing host cell death and feeding on dead tissues. Thanks to an arsenal of plant cell wall-degrading enzymes, to

* Corresponding Author

Host - Pathogen Interaction: Microbial Metabolism, Pathogenicity and Antiinfectives, First Edition. Edited by Gottfried Unden, Eckhard Thines, and Anja Schüfler.

๑) 2016 Wiley-VCH Verlag GmbH \& Co. KGaA. Published 2016 by Wiley-VCH Verlag GmbH \& Co. KGaA. 


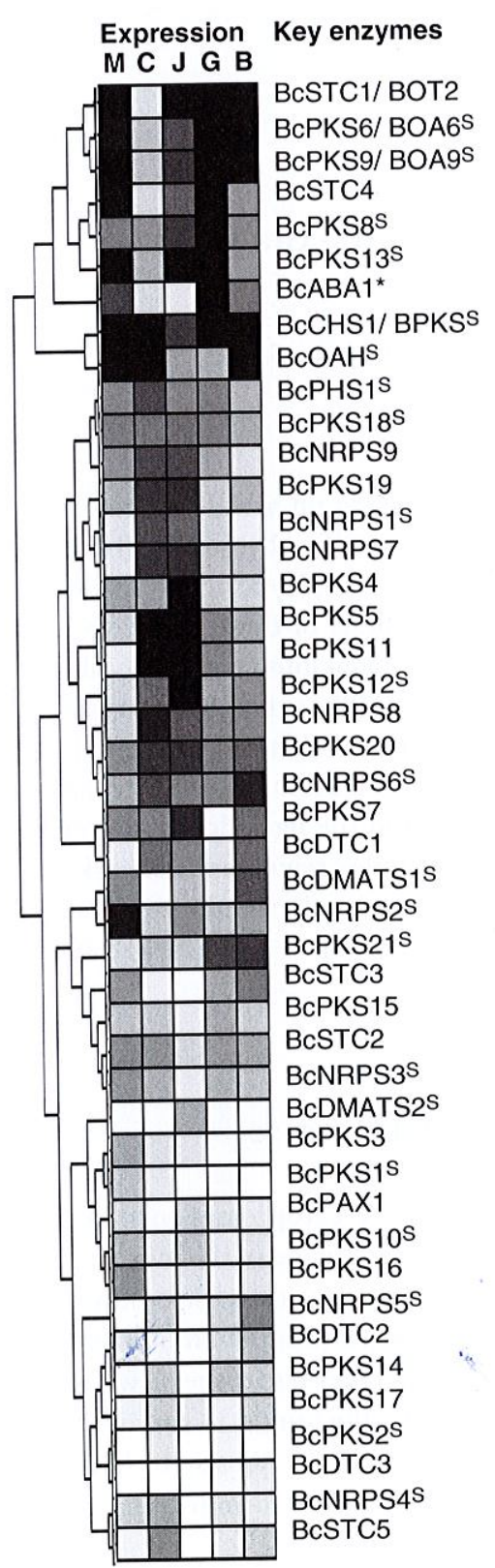

Known metabolites (in bold) or predicted ones

Botrydial (BOT) [13]

Botcinic acid, botcinins (BOA) [14-15]

Botcinic acid, botcinins (BOA) [14-15]

Sesquiterpene [5]

Reduced polyketide [16]

(Conidial) DHN melanin [17-19]

Abscisic acid $[12,20]$

Pyrones, resorcylic acids, resorcinols [21]

Oxalic acid [22]

Carotenoids and retinal [17]

Polyketide [16]

Peptide [23]

Polyketide [16]

Peptide [23]

Ferrichrome siderophore [23]

Reduced polyketide [16]

Amino-acid containing polyketide [16]

Reduced polyketide [16]

(Sclerotial) DHN melanin [17, 19]

Peptide [23]

Polyketide [16]

Coprogene siderophore [23]

Amino-acid containing polyketide [16]

Diterpene [5]

Alkaloid [5]

Ferrichrome siderophore [23]

Polyketide [5]

Sesquiterpene [5]

Polyketide, probably orsellinic acid [24]

Sesquiterpene [5]

Ferrichrome siderophore [23]

Alkaloid [5]

Amino-acid containing polyketide [16]

Reduced polyketide [16]

Indole-diterpene [5]

Reduced polyketide [16]

Polyketide [16]

Peptide [23]

Diterpene [5]

Polyketide [16]

Polyketide [16]

Reduced polyketide [16]

Diterpene [5]

Peptide [23]

Sesquiterpene [5] 
Figure 11.1 Repertoire of the predicted secondary metabolism key enzymes (KE) genes in Botrytis cinerea and their expression in different growth conditions. Conidial suspensions of the wild type strain B05.10 were incubated for $48 \mathrm{~h}$ on minimal medium (M), complete medium $(C)$, grape Juice medium (J), grape berries ( $G$; Vitis vinifera), or on bean leaves (B; Phaseolus vulgaris). Expressions (i.e., log2-normalized intensities) from NimbleGen array data were clustered and depicted by a color scale, where light gray represent weakly expressed genes and dark gray represent highly expressed genes. ${ }^{\mathrm{S}}$ Indicates that the KE is also present in the close species Sclerotinia sclerotiorum [5]. ${ }^{*}$ The KE responsible for ABA synthesis remains unknown, so the gene encoding the BCABA1 P450 monooxygenase was included in the analysis [12].

phytotoxic small proteins and metabolites, and to the ability to encounter strong oxidative stress, the fungus can develop on many different host tissues under a wide range of environmental conditions [3-7]. B. cinerea is more destructive on mature or senescent tissues, but it can gain entry to these tissues at a much earlier stage and remain quiescent until the host physiology becomes favorable for its proliferation [3]. In some cases, the endophytic behavior of B. cinerea was clearly demonstrated $[8,9]$. Finally, under specific environmental conditions in vineyards, $B$. cinerea may not cause gray mold but noble mold, which allows the production of sweet wines like Sauternes. In this case, the development of the fungus is limited to the outermost layers of grape epidermis [10]. In conclusion, B. cinerea encounters many different biotic and abiotic interactions and consequently modifies its metabolism and physiology to adapt to the altering environmental conditions.

\section{Repertoire of Secondary Metabolites Produced by B. cinerea}

Secondary metabolites (SMs) are expected to play an essential role in the versatile life of $B$. cinerea as described in the previous section. In the pregenomic era of this fungus, about eight families of SMs have been isolated from in vitro mycelium, but sequencing of the genome revealed a repertoire of gene clusters dedicated to the synthesis of approximately 40 different SMs $[5,11]$. As in other fungi, these clusters include one (or two) gene(s) encoding the key enzyme (KE) responsible for synthesis of the raw product, genes encoding enzymes for further modifications and in some cases, genes encoding transporters and/or regulators. Among the $44 \mathrm{KEs}$ (Figure 11.1) are many polyketide synthases (21 PKSs) [16, 21, 24], nonribosomal peptide synthetases (9 NRPSs) [23], and sesquiterpene cyclases (5 STCs) and diterpene cyclases (3 DTCs). Notably, only 21 of these KE-encoding genes are shared with the closest related genus Sclerotinia [5]. To date, only a small number of SMs have been identified and connected with the genetic make-up of B. cinerea. Among them are the predominant nonspecific toxins botrydial (BOT) and botcinic acid (BOA) (Figure 11.2) and other SMs that may be involved in plant/fungus interactions:

- Botrydial (BOT) and related compounds are sesquiterpenoids [26]. The discovery of the genetic basis for BOT biosynthesis included the identification of a cluster of coregulated genes [13,27]. Deletion analyses of bcbot1, bcbot3, 
bcbot4 encoding cytochrome P450 monooxygenases and bcbot $2 / b c s t c 1$ encoding a STC proved their requirements for BOT biosynthesis [13, 25] (I.G. Collado et al., unpublished). BOT would trigger plant cell death via induction of the hypersensitive response [28].

- Botcinic acid (BOA) and its derivatives (botcinins) are combined polyketides [29, 30]. The cluster of coregulated genes (bcboa1-17; Figure 11.2) includes two PKSencoding genes essential for BOA biosynthesis: $\mathrm{BCBOA} 6$ mediates the formation of the tetraketide core, whereas $\mathrm{BCBOA} 9$ is responsible for the synthesis of the side chain $[14,15]$. Analysis of mutants lacking the ability to produce BOT, BOA, or both toxins suggested a redundant role in plant tissue colonization [14]. Additionally, several botcinins would have antifungal activities $[29,30]$.

- Abscisic acid ( $A B A)$ is a plant hormone and belongs to the group of sesquiterpenoids [31]. While in higher plants, its biosynthesis is mediated via the carotenoid (CAR) pathway, B. cinerea synthesizes ABA directly from farnesyl diphosphate via different oxidative steps. The $B$. cinerea $\mathrm{KE}$ gene responsible for the first step of ABA biosynthesis remains unknown but a cluster of four genes (bcaba1-4) involved in the later steps was characterized [12, 20]. To date, no evidence exists that fungus-derived $A B A$ influences fungus-host interactions (V. Siewers and P. Tudzynski, unpublished).

- Oxalic acid $(O A)$ is a compound that is produced via the oxaloacetate acetylhydrolase $(\mathrm{OAH})$ in B. cinerea and Sclerotinia sclerotiorum. Loss of OA formation affects virulence in closely related fungi in different ways: while it results in avirulent mutants in S. sclerotiorum, bcoahA mutants are still able to colonize the host tissue $[22,32,33]$.

B. cinerea also produces several pigments that can act as protectants against UV and other exogenous stresses (oxidative stresses, desiccation):

- 1,8-Dihydroxynaphthalene (DHN)-melanin is a polyketide derivative accumulating in the conidiophores, conidia, and sclerotia of $B$. cinerea, giving them their characteristic color [34, 35]. In contrast to other DHN-melanin forming fungi, B. cinerea possesses two developmentally regulated PKS (KE)-encoding genes (bcpks12, bcpks13), while the other melanogenic genes are present in single copies [17]. Recently, it was shown that BcPKS12 and BcPKS13 provide the precursors for sclerotial and conidial melanogenesis, respectively [18, 19].

- Carotenoids (CARs) are tetraterpenoids produced by plants and fungi to protect cells from free radicals and singlet oxygen [36]. A cluster of four light-induced genes (bop2-bcphd1-bcphs 1-bccao'1) was recently identified in B. cinerea [17] that shares similarity with the CAR gene cluster in Fusarium fujikuroi suggesting that B. cinerea produces CAR via a similar biosynthetic pathway than F. fujikuroi [37].

- Bikaverin (BIK), a red pigment, is a well-known polyketide in some Fusarium species [38] but that is also produced by rare strains of $B$. cinerea due to a probable horizontal gene transfer $[39,40]$.

The repertoire of KEs (Figure 11.1) suggests that $B$. cinerea can also produce several siderophores [23] and many other SMs that remain to be chemically identified. 

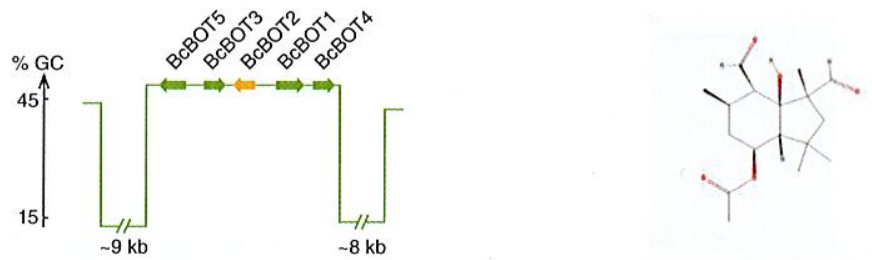

(a) Botrydial (sesquiterpene)

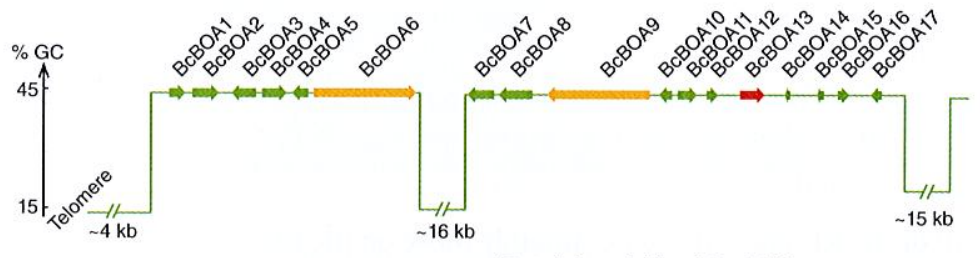

Botcinic acid (polyketide)

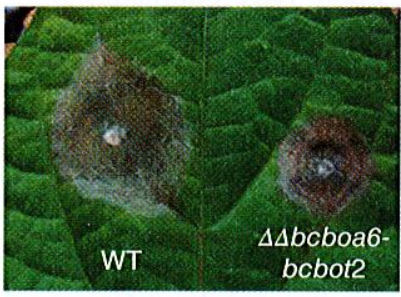

(b)

French bean, 3 dpi .

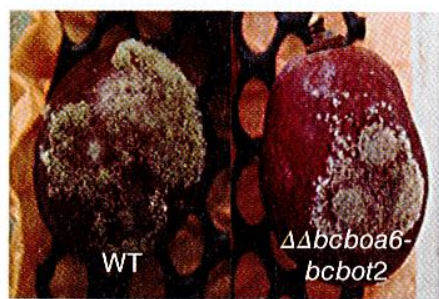

Grape, 10 dpi

Figure 11.2 The two toxins botcinic acid (BOA) dehydrogenases (bcboa5 and 17), a and botrydial (BOT) are produced from clusters FAD-binding protein (bcboa8), a dehydratase of co-regulated genes and have a redundant role in virulence. (a) BOT and BOA clusters (bcboa16), a thioesterase (bcboa 10), a predicted in the wild strain B05.10 $[13,14]$ and surrounding AT-rich regions ( $\mathrm{J}$. van Kan et al., unpublished); chemical structures of BOT and BOA (PubChem). The KE for BOT synthesis is the Sesquiterpene cyclase (STC) encoded by bcbot2/stc1. Other co-regulated genes encode for P450 monooxygenases (bcbot 1, 3, and 4) also involved in BOT synthesis [12] (I. G. Collado et al., unpublished) and a putative acetyl transferase (bcbot5). Two KEs are required for transferase (bcboa 11), and unknown proteins (bcboa12, 14, and 16). A pathway-specific $\mathrm{Zn}(\mathrm{II})_{2} \mathrm{Cys}_{6}$ transcription factor (TF) is encoded by bcboa 13 and a Nmr-A like regulator is putatively encoded by bcboa1. (b) While both single mutants $\Delta b c b o a 6$ and $\Delta b c b o t 2$, impaired in BOA and BOT production, respectively, are not altered in virulence compared to the wild type (WT) strain (not shown), the double $\Delta \Delta b c b o a 6$-bcbot 2 mutant, unable to produce any toxin, causes significant BOA synthesis: the polyketide synthases (PKSs) bcbot6/pks6 and bcboa9/pks $9[14,15]$. Other co-regulated genes encode for putative monooxygenases (bcboa2, 3, 4, and 7), smaller necrotic lesions on several host plants. Here, conidial suspensions were inoculated on leaves of Phaseolus vulgaris (French bean) and Vitis vinifera (grape) berries. 
Importantly, the property to produce the mentioned SMs varies among $B$. cinerea wild strains. ABA and BIK are only formed by few strains $[12,40]$, and not all wild strains produce OA, BOT, and BOA $[41,42]$.

\section{Expression of SM Genes during In Vitro and In Planta Conditions}

In the past years, several genome-wide expression studies were designed to investigate the transcriptome of $B$. cinerea either during saprophytic growth on various culture media or during the infection process on different host plants. Figure 11.1 presents the expression patterns of the SM KE-encoding genes of the model strain B05.10 after 2 days of growth in three in vitro conditions versus two in planta conditions. The media used were minimal medium (M) [43], complete medium (C) [17], and grape juice medium $(\mathbf{J})$ [44]. The in planta conditions are grape berries (G) [43] and bean leaves (B) [41] both at 48-h postinfection. Figure 11.1 highlights several features about the regulation of SM in B. cinerea:

- About half of the KE-encoding genes (mainly those on the top of the Figure 11.1) are highly expressed in at least one of the culture conditions. Among the tested media, the one made with grape juice stimulates the highest number of KE-encoding genes (16 genes highly expressed). This medium with a high sugar content (about $200 \mathrm{gl}^{-1}$ ), a low $\mathrm{pH}$ (around 4), and the presence of plant compounds was originally designed to mimic the conditions that the fungus encounters during infection of grape berries [44] and then chosen to compare the expression of SM genes in different regulation mutants (see Section 11.5 and Figure 11.3).

- In opposite, five of the KE-encoding genes (e.g., bcpks2 or bcdtc3) are not expressed in any condition tested. Whether these genes are functional in $B$. cinerea remains to be elucidated.

- Finally, the clustering shows that BOT and BOA KE-encoding genes have similar expression patterns. This coregulation includes also the other $b c b o t$ and $b c b o a$ genes presented in Figure 11.2. These data indicate that the biosynthesis of both toxins is activated during plant tissue colonization and in grape juice medium but also in synthetic minimal medium. The moderate expression under in vitro conditions has allowed for the identification of the underlying biosynthetic pathways [45].

Overall, the transcriptomic data indicate that, on one hand, the SM genes belonging to one cluster are tightly coregulated $[13,14,20]$ and, on the other hand, almost each cluster has its own expression pattern when we consider the different growth conditions. It is known from other fungi that the regulation of SM gene clusters occurs at several levels, that is, by pathway-specific transcription factors (TFs) that are usually encoded by a cluster gene, by broad domain TFs that integrate environmental signals such as the availability of carbon and nitrogen sources and the ambient $\mathrm{pH}$, and by other global regulators that could affect gene expression via chromatin remodeling [46-48]. Recent advances regarding $B$. cinerea are presented in the following sections. 


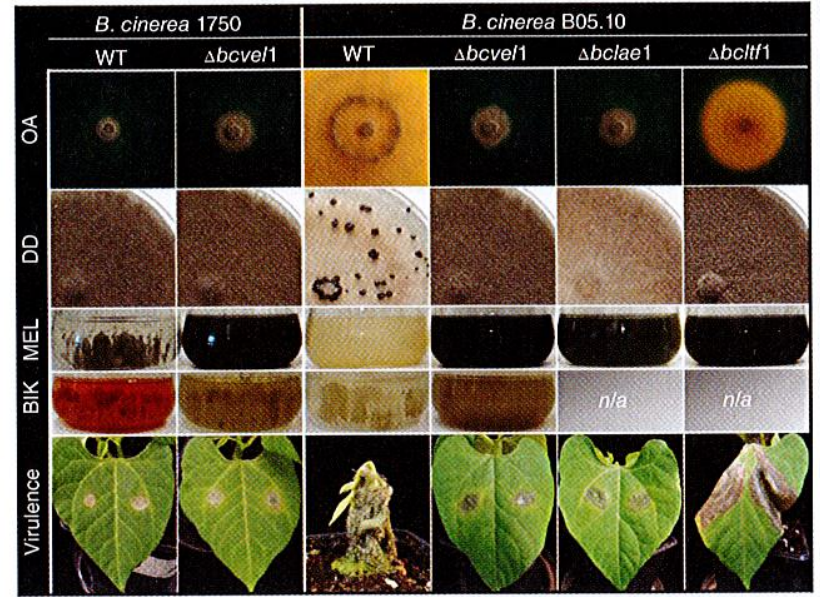

(a)

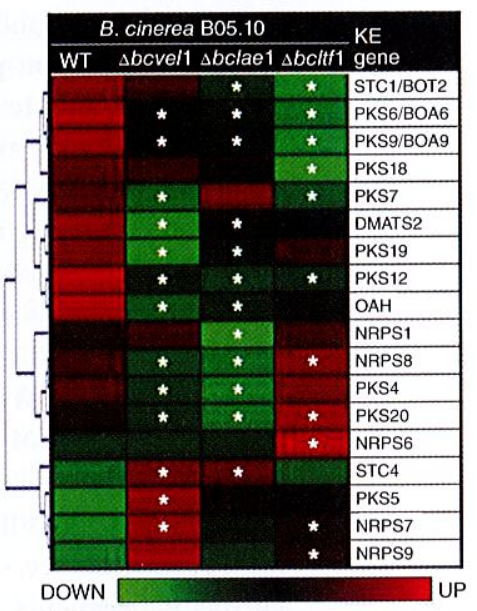

(b)
Figure 11.3 Members of the VELVET complex (BCVEL1, BCLAE1) and the light-responsive transcription factor BCLTF1 control light-dependent differentiation, secondary metabolism, and virulence. (a) Phenotypes of the deletion mutants in wild strain 1750 (BIK producer) and the standard recipient strain B05.10. OA - detection of oxalic formation by acidification of the culture medium $6 \mathrm{dpi}$ (bluish green: $\mathrm{pH}>7$, yellow $\mathrm{pH}<6$ ). DD - differentiation phenotype 12 dpi in constant darkness; the WT:B05.10 produces sclerotia while the other strains produce conidia. MEL - accumulation of DHN melanin in liquid cultures $7 \mathrm{dpi}$ (minimal medium with $\mathrm{NaNO}_{3}$ as nitrogen source). BIK - accumulation of bikaverin in liquid cultures $7 \mathrm{dpi}$ (minimal medium with $\mathrm{NH}_{4} \mathrm{NO}_{3}$ as nitrogen source).

Virulence - lesion formation on primary leaves of Phaseolus vulgaris (French bean) $6 \mathrm{dpi}$. (b) Comparative gene expression studies of the mutants. Conidial suspensions of the wild type B05.10 and the three deletion mutants were incubated for $48 \mathrm{~h}$ on solid grape juice medium with cellophane overlays. Material from four biological replicates were used for hybridization of NimbleGen arrays. Statistical analyses revealed $18 \mathrm{KE}$-encoding genes that are differentially expressed in at least one mutant $\left(^{*}\right.$ : fold change $\left.>2 ; p<0.05\right)$. Relative expression values of these genes (i.e., log2-normalized intensities scaled by gene) were clustered and depicted by color scale, in which shades of green and red represent under- and overexpressed genes, respectively. GEO accession GSE63021 and (A. Simon et al., unpublished).

\section{Pathway-Specific Regulation by Transcription Factors}

Many fungal SM gene clusters contain a gene that encodes a $\mathrm{Zn}$ (II)2Cys6 zinc finger TF that specifically regulates the adjacent biosynthetic genes [48]. In B. cinerea, $40 \%$ of the SM clusters, including the BOA one, contain a gene encoding such a candidate TF $[5,14]$. The function of the candidate bcboa13 (Figure 11.2) was recently investigated by gene deletion and expression analysis. Results indicated that BcBOA13 regulates genes that are part of the BOA cluster but not other SM genes such as those of the BOT cluster, suggesting that BcBOA13 is indeed a BOA-specific TF (A. Porquier et al., unpublished). Another pathway-specific TF identified in $B$. cinerea is the $\mathrm{Zn}(\mathrm{II}) 2 \mathrm{Cys} 6$ protein BcRUM1 (regulator of unknown metabolite). 
Bcrum1 inactivation demonstrated that this TF is required for the co-expression of the four adjacent genes that are all possibly involved in the biosynthesis of an unknown metabolite during the early stages of the infection process [49].

As opposed to the BOA cluster, the predicted BOT cluster lacks a TF-encoding gene (Figure 11.2) [13]. Therefore, a yeast one-hybrid (YOH) approach was developed to identify regulators of the BOT genes [44]. The bidirectional promoter of bcbot1/bcbot 2 was used as bait for screening a library containing 396 out of 406 $B$. cinerea TFs, revealing a physical interaction of the BOT promoter with a Cys2His2 TF (BcYOH1). A global transcriptomic analysis of the $\Delta b c y o h 1$ mutant further revealed that this TF is not specific to the BOT cluster but is rather involved in the regulation of a wide range of genes. The expression of 22 SM clusters including the BOT and BOA ones appeared $\mathrm{BcYOH} 1$ dependent. Moreover, the expression of genes involved in other processes such as carbohydrate metabolism, transport, virulence, or detoxification was also affected in the $\Delta$ bcyoh1 mutant [44]. Further experiments are ongoing to identify a possible BOT-specific TF.

\section{Concerted Regulation of Secondary Metabolism and Light-Dependent Development}

A prominent global regulator of fungal SM is the putative methyltransferase LaeA (loss of aflR expression) that was first identified in Aspergillus nidulans as a regulator of SM gene clusters [50,51]. Later, LaeA was shown to form a protein (VELVET) complex with the fungal regulatory proteins VeA and VelB that coordinates development and secondary metabolism in response to light in A. nidulans and other filamentous ascomycetes [52-54]. Light is also an important environmental cue for B. cinerea as it triggers the formation of macroconidia and represses the formation of sclerotia. Like $A$. nidulans, B. cinerea contains a VELVET complex composed of the orthologs BcVEL1 (VeA), BcVEL2 (VelB), and BcLAE1 (LaeA) whereby BcVEL1 functions as the bridging partner by interacting with both BcVEL2 and BcLAE1 [55]. General features of $\Delta$ bcvel1 and $\Delta$ bclae1 mutants are the "always conidia" phenotype (conidiation in light and darkness accompanied by the loss of sclerotia formation), increased production of conidial/BcPKS13-derived melanin, loss of OA formation, deregulation of several SM-related genes, and reduced virulence on bean and tomato (Figure 11.3) $[41,55]$. The deletion of the VelB ortholog BcVEL2 results in a similar phenotype to that of $\Delta b c v e l 1$ regarding differentiation and virulence [56], which is in accordance with the interaction found between these two proteins. The outstanding role of BcVEL1 as the central part of the VELVET complex that links development and virulence is furthermore supported by the finding that mutations of this gene (single nucleotide polymorphisms causing stop codons) were identified in two wild strains $(\mathrm{T} 4,1750)$ showing the "always conidia" phenotype and reduced aggressiveness on the host $[40,41]$. The latter strain (1750) belongs to the group of the BIK producers; and by targeted deletion of the whole bcvel1 in this genetic background it was shown that the formation of the red pigment depends on the VELVET complex (Figure 11.3) [40]. 
Recently, the light-responsive TF1 (BcLTF1) was identified by a random mutagenesis approach. As deletion mutants of the VELVET complex, the $\Delta$ bcltf1 mutant exhibits hyper-conidiation accompanied by increased production of conidial/BcPKS13-derived melanin and loss of sclerotia formation (Figure 11.3). However, in contrast to the other mutants, $\Delta b$ cltf1 mutants massively suffer from oxidative stress and are therefore severely impaired in growth in the light. Microarray analyses were performed to compare the transcriptional responses to $60 \mathrm{~min}$ of light treatment between undifferentiated mycelia of the wild type and the mutant demonstrating that BcLTF1 is required for proper regulation of the majority of light-responsive genes and additionally for the regulation of SM-related genes in a light-independent manner [17]. However, the question remains open whether BcLTF1 directly affects SM by its function as a TF or whether the effect is indirect and possibly due to changes in primary metabolism and/or the cellular redox status.

For the direct comparison of the impacts of BcVEL1, BcLAE1, and BcLTF1 on gene expression, a microarray approach was performed using grape juice medium and the three deletion mutants (Figure 11.3). Even under this condition, the deletion of $b c l t f 1$ affected the expression profile of $11 \mathrm{KE}$-encoding genes; six genes are underexpressed and five genes are overexpressed compared to the wild type. Bcpks13 is also overexpressed in the mutant, but to a lesser extent than on complete medium [17]. This is because bcpks13 is already strongly expressed in the wild type on grape juice medium compared to the complete medium (Figure 11.1). The deletions of both VELVET complex members affect the expression levels of several KE-encoding genes; the profiles were similar but not identical. Thus, $b c p k s 7$ is underexpressed in $\Delta$ bcvel1 and $\Delta$ bcltf1 but not in $\Delta$ bclae1, and bcnrps 1 is underexpressed in $\Delta$ bclae1 only [55] (J. Schumacher et al., unpublished).

BcWCL1 ("white collar"-like 1) is an example for a broad-domain TF that allows for the integration of light signals due to a blue light-sensing LOV (light-oxygenvoltage) domain. Deletion mutants exhibit hyper-conidiation as well but are not affected in OA formation and virulence in standard illumination conditions. Moreover, the mutants are partially "blind": expression of certain light-responsive genes is no longer induced by light [57]. Recent genome-wide expression analyses revealed that a couple of KE-encoding genes are differentially expressed in the mutant, for example, bcpks13 (conidial melanin) is overexpressed while bcphs 1 (CARs and retinal) is not induced in response to light treatment in the mutant (J. Schumacher et al., unpublished).

The strong interdependence of SM and light-dependent development in B. cinerea is furthermore supported by identification of other mutants that are affected in both processes. For instance, the deletion of the bZIP-TF BcATF1 results in increased accumulation of aerial hyphae, reduced conidiation, loss of sclerotia formation, and increased production of BOA and BOT, which is accompanied by slightly increased colonization efficiencies on different host plants. Though BOA- and BOT-encoding genes are underexpressed in the mutant, no physical interactions between their promoters and BcATF1 could be established by yeast one-hybrid experiments, which suggests an indirect effect on SM-related gene expression [58]. 


\section{Regulation of Secondary Metabolism by Conserved Signal Transduction Pathways}

Another level of regulation of SM is mediated by conserved signal transduction pathways that comprise cAMP (cyclic adenosine monophosphate) and $\mathrm{Ca}^{2+}$ as second messengers and further include Ras superfamily proteins and mitogen-activated protein (MAP) kinases. These pathways may regulate broad domain and pathwayspecific TFs. The key components of the pathways including the three MAP kinases BMP1 (FUS3/KSS1 ortholog), BMP3 (SLT2 ortholog) and BcSAK1 (HOG (high osmolarity glycerol) ortholog), the adenylate cyclase $\mathrm{BAC}$ and the protein kinase A (PKA) that are part of the cAMP cascade, and the calcineurin phosphatase $(\mathrm{CN})$ as one of the main actors of the $\mathrm{Ca}^{2+}$ signaling pathway. Heterotrimeric $\mathrm{G}$ proteins that act as upstream elements have also been functionally studied in $B$. cinerea with regard to development, responses to different kinds of stresses as well as virulence $[59,60]$. However, not much is known how these signaling pathways influence SM. The deletions of the three MAP kinases affect the production of melanin, which is accompanied by defects in proper differentiation of conidia and/or sclerotia [61]. A recent study revealed impact of the stress-activated MAP kinase BCSAK1 on the formation of the known phytotoxins: BOT, BOA, and its derivatives are produced in much lower quantities in axenic cultures of $\Delta b c s a k 1$ mutants compared to the wild type [62]. The same effect was found in deletion mutants of the G $\alpha$ subunit BCG1. As mutants of the cAMP pathway, that is, $\triangle b a c$ and $\triangle b c p k a 1$ mutants are not impaired in the formation of toxins [63], a second downstream pathway of BCG1 has been hypothesized. This turned out to be the $\mathrm{Ca}^{2+} / \mathrm{CN}$ pathway that is linked with the heterotrimeric G protein via a phospholipase C (BCPLC1) [64]. The inhibition of $\mathrm{CN}$ by cyclosporine $\mathrm{A}$ and the deletion of components of the latter pathway such as the $\mathrm{CN}$ regulator $\mathrm{BCRCN1}$ and the $\mathrm{CN}$-responsive TF BCCRZ1 resulted in decreased expression levels of BOT- and BOA-biosynthetic genes [27, 66]. More recently, the contribution of $\mathrm{Ca}^{2+}$-dependent signal transduction to the regulation of the carotenogenic genes has been revealed; their expression levels are induced by $\mathrm{Ca}^{2+}$ addition, suggesting a link between $\mathrm{Ca}^{2+}$ - and light-/BcWCL1-dependent signaling (J. Schumacher et al., unpublished).

\section{Role of the Chromatin Landscape in the Regulation of Secondary}

Active TFs are not always sufficient to express SM genes as clusters can be embedded within transcriptionally silent heterochromatin. Chromatin-based regulation through histone acetylation and methylation has recently been shown to play a significant role in the regulation of SM clusters in A. nidulans and other model fungi $[47,48,67]$. In $B$. cinerea, the occurrence of such mechanisms has not yet been shown, but several relevant clues are already available.

Firstly, some of the clusters are in particular genomic environments. The whole genome sequence of $B$. cinerea was recently assembled into 16 core chromosomes 
(J. van Kan et al., unpublished) and this revealed that six SM gene clusters including the BOA one are located in subtelomeric regions. bcboal is even the closest gene to one of the telomeres of chromosome 1. Studies in other fungi have shown that these regions are more often subjected to chromatin-mediated regulation [67]. In addition, both BOT and BOA clusters are surrounded by several kb of AT-rich regions (>80\%; Figure 11.2) [14] (J. van Kan et al., unpublished). In the case of the BOA cluster, a $16 \mathrm{~kb}$-stretch of AT-rich sequence is even present inside the cluster between bcboa6 and bcboa7. Again, such AT-rich regions may correspond to heterochromatin structure and may be under the control of histone modifiers as demonstrated for effector genes that are specifically expressed during infection in Leptosphaeria maculans [68]. The fact that the clusters encoding the two main phytotoxins of B. cinerea share similar AT-rich surrounding regions raises questions about the importance of the role of these genomic features in chromatin structure and gene expression.

Secondly, the characterization of several regulators in B. cinerea revealed a possible involvement of chromatin-mediated regulation. The mode of action of the global regulator LaeA mentioned above remains enigmatic, but they are some evidences in A. nidulans that it counteracts some silencing heterochromatic marks [69].

Another example is the transcriptional regulator BcREG1 that is required for conidiogenesis, production of BOA and BOT, and colonization of the host tissues [70]. The mode of action of BcREG1 remains obscure, but a recent study on the ortholog SGE1 in Fusarium fujikuroi suggests that these regulators may act on the chromatin level [71].

Finally, the mutation of bcspt3, encoding a component of the multisubunit SAGA (Spt-Ada-Gcn5-acetyltransferase) complex, resulted in an altered brownish/yellowish pigmentation suggesting that the disruption of the SAGA complex may affect the repertoire of expressed SM genes in B. cinerea [72]. The SAGA complex is required to activate transcription of a subset of RNA polymerase IIdependent genes by modifying the histones, especially in response to environmental stresses $[73,74]$.

Genetic studies on histone modifiers are currently in progress to investigate the role of chromatin modifications (methylation and acetylation) in the regulation of secondary metabolism in B. cinerea (J. Schumacher et al., unpublished; A. Porquier et al, unpublished).

\section{Concluding Remarks}

Although many actors of the regulation of the secondary metabolism in B. cinerea have been identified in the past years, important studies remain to be conducted to understand the complex regulatory network from the upstream environmental signals to the downstream regulators that directly interact with SM biosynthetic gene clusters to allow the production of bioactive compounds. This knowledge is crucial to understand the development and virulence of $B$. cinerea and may provide useful start points for the identification of new biological targets to control 
this crop-devastating fungus. This knowledge could also provide approaches to "wake up" silent SM clusters during in vitro growth and discover new bioactive compounds. Possible approaches include genetic engineering (e.g., overexpression of pathway-specific TFs, modification of the chromatin landscape) and co-culturing with other microorganisms $[47,74]$. In addition to their activities toward plants, new SMs isolated from $B$. cinerea could have interesting toxic activities toward other organisms (fungi, oomycetes, insects, bacteria, nematodes, etc.) that can be exploited in biotechnology. Indeed, as more fungal genome sequences are becoming available and more SM biosynthetic genes are being identified, fungi re-emerge as important resources for new therapeutic and agrochemical agents $[75,76]$.

\section{References}

1 Dean, R. et al. (2012) The top 10 fungal pathogens in molecular plant pathology. Mol. Plant Pathol., 13 (4), 414-430.

2 Coley-Smith, J.R., Verhoeff, K., and Jarvis, W.R. (1980) The Biology of Botrytis cinerea, Academic Press, London.

3 Williamson, B. et al. (2007) Botrytis cinerea: the cause of grey mould disease. Mol. Plant Pathol., 8 (5), 561-580.

4 Choquer, M. et al. (2007) Botrytis cinerea virulence factors: new insights into a necrotrophic and polyphageous pathogen. FEMS Microbiol. Lett., 277 (1), $1-10$.

5 Amselem, J. et al. (2011) Genomic analysis of the necrotrophic fungal pathogens Sclerotinia sclerotiorum and Botrytis cinerea. PLoS Genet., 7 (8), e1002230.

6 van Kan, J.A. (2006) Licensed to kill: the lifestyle of a necrotrophic plant pathogen. Trends Plant Sci., 11 (5), 247-253.

7 Hahn, M., Viaud, M., and van Kan, J. (2014) in Genomics of Plant-Associated Fungi and Oomycetes (eds R. Dean, A. Lichens-Park, and C. Kole), SpringerVerlag, Berlin, Heidelberg, pp. 19-44.

8 Barnes, S.E. and Shaw, M.W. (2003) Infection of commercial hybrid primula seed by botrytis cinerea and latent disease spread through the plants. Phytopathology, 93 (5), 573-578.

9 van Kan, J.A., Shaw, M.W., and Grant-Downton, R.T. (2014) Botrytis species: relentless necrotrophic thugs or endophytes gone rogue? Mol. Plant Pathol., 15 (9), 957-961.
10 Pucheu-Planté, B. and Mercier, M. (1983) Etude ultrastructurale de l'interrelation hôte-parasite entre le raisin et le champignon Botrytis cinerea: exemple de la pourriture noble en Sauternais. Can. J. Bot., 61 (6), 1785-1797.

11 Collado, I.G. and Viaud, M. (2016) Secondary metabolism in Botrytis cinerea: combining genomic and metabolomic approaches, in Botrytis - The Fungus, the Pathogen and Its Management in Agricultural Systems (eds S. Fillinger and Y. Elad), Springer, International Publishing, pp. $291-313$.

12 Siewers, V., Smedsgaard, J., and Tudzynski, P. (2004) The P450 monooxygenase BcABA1 is essential for abscisic acid biosynthesis in Botrytis cinerea. Appl. Environ. Microbiol., 70 (7), 3868-3876.

13 Pinedo, C. et al. (2008) Sesquiterpene synthase from the botrydial biosynthetic gene cluster of the phytopathogen Botrytis cinerea. ACS Chem. Biol., 3 (12), $791-801$.

14 Dalmais, B. et al. (2011) The Botrytis cinerea phytotoxin botcinic acid requires two polyketide synthases for production and has a redundant role in virulence with botrydial. Mol. Plant Pathol., 12 (6), 564-579.

15 Massaroli, M. et al. (2013) A shared biosynthetic pathway for botcinins and botrylactones revealed through gene deletions. ChemBioChem, 14 (1), 132-136. 
16 Kroken, S. et al. (2003) Phylogenomic analysis of type I polyketide synthase genes in pathogenic and saprobic ascomycetes. Proc. Natl. Acad. Sci. U.S.A., 100 (26), 15670-15675.

17 Schumacher, J. et al. (2014) The transcription factor BcLTF1 regulates virulence and light responses in the necrotrophic plant pathogen Botrytis cinerea. PLoS Genet., 10 (1), e1004040. Zhang, C. et al. (2015) Loss of bcbrn1 and bcpks13 in Botrytis cinerea not only blocks melanization but also increases vegetative growth and virulence. Mol. Plant-Microbe Interact., 28 (10), 1091-1101

19 Schumacher, J. (2015) DHN melanin biosynthesis in the plant pathogenic fungus Botrytis cinerea is based on two developmentally regulated key enzyme (PKS)-encoding genes. . Mol Microbiol. (in press) doi: 10.1111/mmi.13262.

20 Siewers, V. et al. (2006) Identification of an abscisic acid gene cluster in the grey mold Botrytis cinerea. Appl. Environ. Microbiol., 72 (7), 4619-4626.

21 Jeya, M. et al. (2012) The Botrytis cinerea type III polyketide synthase shows unprecedented high catalytic efficiency toward long chain acyl-CoAs. Mol. Biosyst., 8 (11), 2864-2867.

22 Han, Y. et al. (2007) Oxaloacetate hydrolase, the $\mathrm{C}-\mathrm{C}$ bond lyase of oxalate secreting fungi. J. Biol. Chem., 282 (13), 9581-9590.

23 Bushley, K.E. and Turgeon, B.G. (2010) Phylogenomics reveals subfamilies of fungal nonribosomal peptide synthetases and their evolutionary relationships. BMC Evol. Biol., 10, 26.

24 Jorgensen, S.H. et al. (2014) Fusarium graminearum PKS14 is involved in orsellinic acid and orcinol synthesis. Fungal Genet. Biol., 70, 24-31.

25 Siewers, V. et al. (2005) Functional analysis of the cytochrome P450 monooxygenase gene bcbotl of Botrytis cinerea indicates that botrydial is a strain-specific virulence factor. $\mathrm{Mol}$. Plant-Microbe Interact., 18 (6), 602-612.

26 Collado, I.G., Sanchez, A.J., and Hanson, J.R. (2007) Fungal terpene metabolites: biosynthetic relationships and the control of the phytopathogenic fungus
Botrytis cinerea. Nat. Prod. Rep., 24 (4), 674-686.

27 Viaud, M. et al. (2003) Cyclophilin A and calcineurin functions investigated by gene inactivation, cyclosporin $\mathrm{A}$ inhibition and cDNA arrays approaches in the phytopathogenic fungus Botrytis cinerea. Mol. Microbiol., 50 (5), $1451-1465$.

28 Rossi, F.R. et al. (2011) The sesquiterpene botrydial produced by Botrytis cinerea induces the hypersensitive response on plant tissues and its action is modulated by salicylic acid and jasmonic acid signaling. Mol. Plant-Microbe Interact., 24 (8), 888-896.

29 Tani, H. et al. (2005) Botcinins A, B, C, and $\mathrm{D}$, metabolites produced by Botrytis cinerea, and their antifungal activity against Magnaporthe grisea, a pathogen of rice blast disease. J. Nat. Prod., 68, 1768-1772.

30 Tani, H. et al. (2006) Botcinins E and F and botcinolide from Botrytis cinerea and structural revision of botcinolides. $J$. Nat. Prod., 69, 722-725.

31 Oritani, T. and Kiyota, H. (2003) Biosynthesis and metabolism of abscisic acid and related compounds. Nat. Prod. Rep., 20 (4), 414-425.

32 Liang, X. et al. (2015) Oxaloacetate acetylhydrolase gene mutants of Sclerotinia sclerotiorum do not accumulate oxalic acid, but do produce limited lesions on host plants. Mol. Plant Pathol., 16 (6), 559-571.

33 Stefanato, F.L. et al. (2008) Oxaloacetate acetylhydrolase is responsible for oxalic acid production in Botrytis cinerea and required for lesion expansion on some, but not on most host plants. 3rd Botrytis Genome Workshop, Tenerife, Spain.

34 Doss, R.P. et al. (2003) Melanin in the extracellular matrix of germlings of Botrytis cinerea. Phytochemistry, 63 (6), 687-691.

35 Zeun, R. and Buchenauer, H. (1985) Effect of tricyclazole on production and melanin contents of sclerotia of Botrytis cinerea. Phytopathol. Z., 112, 259-267.

36 Gao, Q. and Garcia-Pichel, F. (2011) Microbial ultraviolet sunscreens. Nat. Rev. Microbiol., 9 (11), 791-802. 
37 Avalos, J. and Estrada, A.F. (2010) Regulation by light in Fusarium. Fungal Genet. Biol., 47 (11), 930-938.

38 Wiemann, P. et al. (2009) Biosynthesis of the red pigment bikaverin in Fusarium fujikuroi: genes, their function and regulation. Mol. Microbiol., 72 (4), 931-946.

39 Campbell, M.A., Rokas, A., and Slot, J.C. (2012) Horizontal transfer and death of a fungal secondary metabolic gene cluster. Genome Biol. Evol., 4 (3), 289-293.

40 Schumacher, J. et al. (2013) A functional bikaverin biosynthesis gene cluster in rare strains of Botrytis cinerea is positively controlled by VELVET. PLoS One, 8 (1), e53729.

41 Schumacher, J. et al. (2012) Natural variation in the VELVET gene bcvel1 affects virulence and light-dependent differentiation in Botrytis cinerea. PLoS One, 7 (10), e47840.

42 Reino, J.L. et al. (2004) Virulence-toxin production relationship in isolates of the plant pathogenic fungus Botrytis cinerea. J. Phytopathol., 152, 563-566.

43 Kelloniemi, J. et al. (2015) Analysis of the molecular dialogue between gray mold (Botrytis cinerea) and grapevine (Vitis vinifera) reveals a clear shift in defense mechanisms during berry ripening. Mol. Plant-Microbe Interact., 28 (11), 1167-1180

44 Simon, A. et al. (2013) Screening of a Botrytis cinerea one-hybrid library reveals a Cys2His2 transcription factor involved in the regulation of secondary metabolism gene clusters. Fungal Genet. Biol., 52, 9-19.

45 Collado, I.G. et al. (2000) Botrytis species: an intriguing source of metabolites with wide ranges of biological activities. Structure,chemistry and bioactivity of metabolites isolated from Botrtyis species. Curr: Org. Chem., 4, 1261-1286.

46 Hoffmeister, D. and Keller, N.P. (2007) Natural products of filamentous fungi: enzymes, genes, and their regulation. Nat. Prod. Rep., 24 (2), 393-416.

47 Brakhage, A.A. (2013) Regulation of fungal secondary metabolism. Nat. Rev. Microbiol., 11 (1), 21-32.
48 Wiemann, P. and Keller, N.P. (2014) Strategies for mining fungal natural products. J. Ind. Microbiol. Biotechnol., 41 (2), 301-313.

49 Leroch, M. et al. (2013) Transcriptome profiling of Botrytis cinerea conidial germination reveals upregulation of infection-related genes during the prepenetration stage. Eukaryot. Cell, 12 (4), 614-626.

50 Bok, J.W. et al. (2005) LaeA, a regulator of morphogenetic fungal virulence factors. Eukaryot. Cell, 4 (9), 1574-1582.

51 Bok, J.W. and Keller, N.P. (2004) LaeA, a regulator of secondary metabolism in Aspergillus spp. Eukaryot. Cell, 3 (2), 527-535.

52 Bayram, O. et al. (2008) VelB/VeA/LaeA complex coordinates light signal with fungal development and secondary metabolism. Science, 320 (5882), 1504-1506.

53 Bayram, O. and Braus, G.H. (2012) Coordination of secondary metabolism and development in fungi: the velvet family of regulatory proteins. FEMS Microbiol. Rev., 36 (1), 1-24.

54 Sarikaya-Bayram, O. et al. (2015) One Juliet and four Romeos: VeA and its methyltransferases. Front. Microbiol., 6, 1 .

55 Schumacher, J. et al. (2015) The VELVET complex in the gray mold fungus Botrytis cinerea: impact of BcLAE1 on differentiation, secondary metabolism, and virulence. Mol. Plant-Microbe Interact., 28 (6), 659-674.

56 Yang, Q., Chen, Y., and Ma, Z. (2013) Involvement of BcVeA and BcVelB in regulating conidiation, pigmentation and virulence in Botrytis cinerea. Fungal Genet. Biol., 50, 63-71.

57 Canessa, P. et al. (2013) Assessing the effects of light on differentiation and virulence of the plant pathogen Botrytis cinerea: characterization of the white collar complex. PLoS One, 8 (12), e84223.

58 Temme, N. et al. (2012) BcAtf1, a global regulator, controls various differentiation processes and phytotoxin production in Botrytis cinerea. Mol. Plant Pathol., 13 (7), 704-718. 
59 Schumacher, J. and Tudzynski, P. (2012) in Morphogenesis and Pathogenicity in Fungi (eds J. Pérez-Martín and A. Di Pietro), Springer-Verlag, Berlin, Heidelberg, pp. 225-241.

60 Schumacher, J. (2016) Signal transduction cascades regulating differentiation and virulence in Botrytis cinerea, in Botrytis - The Fungus, the Pathogen and Its Management in Agricultural Systems (eds S. Fillinger and Y. Elad), Springer International Publishing, pp. 247-267.

61 Liu, W. et al. (2011) The osmosensing signal transduction pathway from Botrytis cinerea regulates cell wall integrity and MAP kinase pathways control melanin biosynthesis with influence of light. Fungal Genet. Biol., 48 (4), $377-387$.

62 Heller, J. et al. (2012) The mitogenactivated protein kinase BcSak1 of Botrytis cinerea is required for pathogenic development and has broad regulatory functions beyond stress response. Mol. Plant-Microbe Interact., 25 (6), 802-816.

63 Schumacher, J. et al. (2008) The cAMPdependent signaling pathway and its role in conidial germination, growth, and virulence of the gray mold Botrytis cinerea. Mol. Plant-Microbe Interact., 21 (11), 1443-1459.

64 Schumacher, J. et al. (2008) The Galpha subunit BCG1, the phospholipase $\mathrm{C}$ (BcPLC1) and the calcineurin phosphatase co-ordinately regulate gene expression in the grey mould fungus Botrytis cinerea. Mol. Microbiol., 67 (5), 1027-1050.

65 Harren, K., Schumacher, J., and Tudzynski, B. (2012) The $\mathrm{Ca} 2+$ /calcineurin-dependent signaling pathway in the gray mold Botrytis cinerea: the role of calcipressin in modulating calcineurin activity. PLoS One, 7 (7), e41761.

66 Schumacher, J., de Larrinoa, I.F., and Tudzynski, B. (2008) Calcineurinresponsive zinc finger transcription factor CRZ1 of Botrytis cinerea is required for growth, development, and full virulence on bean plants. Eukaryot. Cell, 7 (4), 584-601.
67 Galazka, J.M. and Freitag, M. (2014) Variability of chromosome structure in pathogenic fungi--of 'ends and odds'. Curr: Opin. Microbiol., 20, 19-26.

68 Soyer, J.L. et al. (2014) Epigenetic control of effector gene expression in the plant pathogenic fungus Leptosphaeria maculans. PLoS Genet., 10 (3), e1004227.

69 Reyes-Dominguez, Y. et al. (2010) Heterochromatic marks are associated with the repression of secondary metabolism clusters in Aspergillus nidulans. Mol. Microbiol., 76 (6), 1376-1386.

70 Michielse, C.B. et al. (2011) The Botrytis cinerea Reg1 protein, a putative transcriptional regulator, is required for pathogenicity, conidiogenesis, and the production of secondary metabolites. Mol Plant Microbe Interact., 24 (9), 1074-1085.

71 Michielse, C.B. et al. (2015) The global regulator FfSge1 is required for expression of secondary metabolite gene clusters but not for pathogenicity in Fusarium fujikuroi. Environ Microbiol., 17 (8), 2690-2708.

72 Giesbert, S. et al. (2012) Identification of pathogenesis-associated genes by $\mathrm{T}$ DNA-mediated insertional mutagenesis in Botrytis cinerea: a type $2 \mathrm{~A}$ phosphoprotein phosphatase and an SPT3 transcription factor have significant impact on virulence. Mol. Plant-Microbe Interact., 25 (4), 481-495.

73 Baker, S.P. and Grant, P.A. (2007) The SAGA continues: expanding the cellular role of a transcriptional co-activator complex. Oncogene, 26 (37), 5329-5340.

74 Nutzmann, H.W. et al. (2011) Bacteriainduced natural product formation in the fungus Aspergillus nidulans requires Saga/Ada-mediated histone acetylation. Proc. Natl. Acad. Sci. U.S.A., 108 (34), $14282-14287$.

75 Scherlach, K. and Hertweck, C. (2009) Triggering cryptic natural product biosynthesis in microorganisms. Org. Biomol. Chem., 7 (9), 1753-1760.

76 Aghcheh, R.K. and Kubicek, C.P. (2015) Epigenetics as an emerging tool for improvement of fungal strains used in biotechnology. Appl. Microbiol. Biotechnol., 99 (15), 6167-6181. 


\section{The Editors}

Volume Editors:

\section{Prof. Dr. Gottfried Unden}

University of Mainz

Institute for Microbiology and Wine

Research, Johann-Joachim-Becherweg 15 55128 Mainz

Germany

unden@uni-mainz.de

\section{Prof. Dr. Eckhard Thines}

University of Mainz

Institute for Microbiology and Wine

Research, Johann-Joachim-Becherweg 15

55128 Mainz

Germany

thines@uni-mainz.de

\section{Dr. Anja Schüffler}

Institute of Biotechnology and Drug Research, Erwin-Schrödinger-Stra $\beta$ e 2 67663 Kaiserslautern

Germany

schueffler@ibwf.de

\section{Series Editor:}

\section{Prof. Dr. Paul M. Selzer}

Boehringer Ingelheim Animal Health $\mathrm{GmbH}$

Global Pharamceutical R\&D AH

Binger Straße 173

55216 Ingelheim am Rhein

Germany

paul.selzer@boehringer-ingelheim.com

and

University of Tübingen

Interfaculty Institute for Biochemstry . Tübingen

Germany

Wellcome Trust Centre for Molecular Parasitology, Institute of Infection Immunity and Inflammation Faculty of Biomedical \& Life Sciences University of Glasgow

Glasgow

United Kingdom

Cover legend

See page IX
All books published by Wiley-VCH are carefully produced. Nevertheless, authors, editors, and publisher do not warrant the information contained in these books, including this book, to be free of errors. Readers are advised to keep in mind that statements, data, illustrations, procedural details or other items may inadvertently be inaccurate.

Library of Congress Card No.: applied for

\section{British Library Cataloguing-in-Publication Data}

A catalogue record for this book is available from the British Library.

\section{Bibliographic information published by the Deutsche Nationalbibliothek \\ The Deutsche Nationalbibliothek lists this publication in the Deutsche Nationalbibliografie; detailed bibliographic data are available on the Internet at <http://dnb.d-nb.de $>$.}

(c) 2016 Wiley-VCH Verlag GmbH \& Co. KGaA, Boschstr. 12, 69469 Weinheim, Germany

All rights reserved (including those of translation into other languages). No part of this book may be reproduced in any form - by photoprinting, microfilm, or any other means - nor transmitted or translated into a machine language without written permission from the publishers. Registered names, trademarks, etc. used in this book, even when not specifically marked as such, are not to be considered unprotected by law.

Print ISBN: 978-3-527-33745-3

ePDF ISBN: 978-3-527-68239-3

ePub ISBN: 978-3-527-68241-6

Mobi ISBN: 978-3-527-68240-9

oBook ISBN: 978-3-527-68238-6

Cover Design Adam-Design, Weinheim, Germany

Typesetting SPi Global, Chennai, India Printing and Binding Markono Print Media Pte Ltd, Singapore

Printed on acid-free paper 